\title{
Investigation of the operation mode at Ruse-Danube Bridge border checkpoint considered to be a mass service system with incoming flow of automobiles at a non-stationary mode of operation
}

\author{
Dimitar Grozev ${ }^{1 *}$, Velizara Pencheva ${ }^{1}$, Ivan Georgiev $^{2}$, and Ivan Beloev ${ }^{1}$ \\ ${ }^{1}$ Ruse University “Angel Kanchev”, Transport Department, Bulgaria \\ ${ }^{2}$ Ruse University “Angel Kanchev”, Natural sciences and education Department, Bulgaria
}

\begin{abstract}
The present work reviews the Ruse-Danube Bridge border checkpoint and the work carried out for a year, by examining the average daily incoming flows of cargo vehicles. On the basis of the information collected, the daily irregularity of the work at the Ruse-Danube Bridge border checkpoint has been assessed and the system has been modelled as a mass service system to assess capacities in existing infrastructure and organization of work. The type of incoming flow has been specified as a non-stationary Poisson flow. Under non-stationary conditions, the basic values of the system parameters have been calculated and a MatLab application has been created. A system of differential-algebraic equations has been selected for a model according to Kolmogorov model for stochastic processes.
\end{abstract}

\section{Introduction}

Transport is an important element of the international trade activities of a country. The integration of the country into the European space has led to a significant increase in the traffic on the main road sections. The efficient sustainable integration of the national infrastructure into the European one aims to improve the cohesion and the connections between the Republic of Bulgaria and the remaining EU member-states. Besides, it poses new challenges in terms of the completion, maintenance and optimization of our roads. [1-3].

The relatively high share of road transport in the volume of transport work on import and export as well as the indicated increase in the volume of transport work was the prerequisite for the envisaged increase in the number of road border checkpoints during the preparation period for accession of the country to the EU [4].

Border checkpoints are detached territories with a special escape and protection regime, through which only the crossing of the state border is allowed, unless otherwise provided in an international treaty.

By 2018 there are 37 border checkpoints in Bulgaria. The total number of points for heavy goods vehicles is 21 [5].

There are usually large queues of trucks at the border checkpoints. The reasons are different: poor interaction between the internal services; poor international interaction with the respective neighboring state or with a third country; insufficient throughput of border checkpoints; poor organization of work; in some cases, not using capacity capabilities in the border checkpoints; technical reasons, e.g. problems in information systems, etc.

Assessing capacity capabilities at border checkpoints may provide preliminary information on the possible number of cars processed and waiting times for available infrastructure and work experience. The lack of methodology for such an assessment does not allow for pre-planning. In this publication, on the basis of collected information, the seasonal inconsistency of work at the border checkpoints is assessed and their mass service system work is modelled. The time for vehicles to stay at different incoming flows, as well as the limit value of the number of cars that can be handled is determined.

The main purpose of this publication is to research the incoming flow of a four channel mass service system and to establish its effectiveness. The following tasks have been set:

- input modelling;

- defining the type of mass service system;

- solving the system of differential-algebraic equation [6-8].

\section{Exposition}

In order to describe the operating regime of Rousse border checkpoints, considered as a mass service system, it is necessary to know the characteristics of the incoming flow of trucks considered as stochastic process, the intensity of service and the number of service units $[9,10]$.

For the incoming flow of trucks, we can set the following prerequisites:

\footnotetext{
${ }^{*}$ Corresponding author: dgrozev@uni-ruse.bg
} 
- ordinary flow - the probability of two or more trucks entering the system for an elementary time interval is infinitesimal, compared to the probability of only one truck doing it; i.e. the property of ordinariness means that automobiles enter one at a time rather than two or three, etc.

- flow without consequences - the number of trucks arriving in the system for time interval $\Delta t$ does not depend on how many vehicles have already been received, that is, does not depend on the history of the studied phenomenon (the flow without action afterwards (Poisson flow);

- stationarity / non-stationarity of the flow [11-12] - for sufficiently long periods of time - 1 month, 6 months, 1 year, etc. it is possible to assume a steady-state of the incoming stream, that is to say, with certain conventions. The probability of occurrence of a certain number of cars in a given, sufficiently long interval depends only on the length of that interval. Generally, at random times, flow $\lambda$ is non-stationary $\lambda=\lambda(t)$. This non-stationarity is well marked for 1 business day (24 hours).

The incoming and outgoing stream is considered and modelled. Depending on the measurements and observations made, an approximation function of the input flow density is determined. The same applies to service speed (outflow).

Table 1 shows the number of trucks waiting in the queue, as well as the number of arrivals each hour.

Table 1. Number of trucks waiting in the queue for two randomly selected days.

\begin{tabular}{|c|c|c|}
\hline $\begin{array}{c}\text { Time } \\
\text { interval, } \mathrm{h}\end{array}$ & $\begin{array}{c}\text { Number of trucks in } \\
\text { the queue on 5.7.2018 }\end{array}$ & $\begin{array}{c}\text { Number of trucks in } \\
\text { the queue on 17.7.2018 }\end{array}$ \\
\hline $0-2$ & 74 & 69 \\
\hline $2-4$ & 22 & 28 \\
\hline $4-6$ & 11 & 15 \\
\hline $6-8$ & 6 & 9 \\
\hline $8-10$ & 3 & 4 \\
\hline $10-12$ & 7 & 5 \\
\hline $12-14$ & 14 & 13 \\
\hline $14-16$ & 26 & 25 \\
\hline $16-18$ & 53 & 49 \\
\hline $18-20$ & 88 & 90 \\
\hline $20-22$ & 107 & 102 \\
\hline $22-24$ & 93 & 89 \\
\hline
\end{tabular}

Concerning the intensity of servicing trucks, according to the Head of the Border Checkpoint, the service time of one truck is a constant value and is 7 minutes (scheduled service time), $\mu=$ const.

Regarding the number of serving channels (servers), it can, if necessary, reach $5(1 \leq s \leq 5)$. In order to provide 24 hours of continuous work per channel, three teams are required (one every 8 hours). For system performance testing, it is necessary to determine the probability that the system will have $k$ automobiles at a moment $t$ with $s$ running servers $[13,14]$ :

$$
P_{k}(t)=?, k=\overline{0, \infty}, t \in[0, T], 1 \leq s \leq 5,
$$

where for a single period $T$, a full working day $T=1$ of 24 hours was taken. The beginning of the working day $t=0$ coincides with the astronomical start of the day ( 0 hours and 0 minutes), the end of the working day $t=T$, with the end of the astronomical day ( 24 hours and 0 minutes).

For the mass service system model, the following can be summarized as follows: a non-stationary stream of requests with density $\lambda(t)$ enters a mass service with $\mathrm{s}$ service channels. Request service time is a random variable with an indicative distribution and parameter $\mu=$ const. A car that arrives at a busy channel time stands in the waiting line and "patiently" waits to be served, that is, a system with unlimited waiting and an unlimited number of trucks in the queue.

Given the characteristics of the incoming and outgoing flow, as well as the behavior of the system itself (multichannel system without failures, endless queuing time, behavior in the "first arrived" queue, etc.), a specific type of differential equation system (of Kolmogorov) is applied. The probabilities of having a certain number of automobiles in the system at any given moment represent solutions to the differential equation system. From these probabilities, predictions are made for the waiting time of a vehicle arriving at a specific time of the day, the number of cars in the system at that time, and other characteristics. The theoretical results are compared with the measurements. If necessary, the functions that describe the incoming and outgoing stream are corrected.

From what has been said so far, we can say that the system is of type $(\mathrm{M} / \mathrm{M} / \mathrm{s})$ in non-stationary mode. To describe a system of this type, the following system of differential equations of Kolmogorov (ErlangKolmogorov) is in effect [15-16]:

$$
\begin{gathered}
\frac{d P_{0}(t)}{d t}=-\lambda(t) P_{0}(t)+\mu P_{1}(t) \\
\ldots \ldots \\
\frac{d P_{i}(t)}{d t}=\lambda(t) P_{i-1}(t)-(\lambda(t)+i \mu) P_{i}(t)+(i+1) \mu P_{i+1}(t) \\
\frac{d P_{s+n}(t)}{d t}=\lambda(t) P_{s+n-1}(t)-(\lambda(t)+s \mu) P_{s+n}(t)+s \mu P_{s+n+1}(t) \\
1 \leq i \leq s-1, n=\overline{0, \infty} .
\end{gathered}
$$

$n$ indicates the number of cars in the queue when all servers are busy. The system of differential equations is infinite and for the numerical solution it is necessary to take a finite number of equations. The number of equations is such that from a given number $N$, the sum $\sum_{i=N}^{\infty} P_{i}(t)$ must not exceed a given number $e$. In this paper, a system of $N=505$ differential equations are solved, with a system "slicing" error less than $\varepsilon=10^{-8}$. After selecting a finite number of equations, the number of probabilities $P_{k}(t)$ is one less than the number of equations, so it is necessary to introduce the additional algebraic equation for normality $\sum_{i=0}^{N-1} P_{i}(t)=1$. The computational features associated with a system (2) after "cutting" and introducing an algebraic equation are as follows:

- the system is of great size;

- the system is differential-algebraic;

- the system is of the "rigid system" type.

This can be summarized as: a system of solid, differential-algebraic equations with a large dimension. Numerical methods have been developed to overcome these difficulties. A MatLab program was developed to 
solve a system (2), using the built-in "solver" ode15s $[15,20]$, implementing the Gir method. When entering $\lambda(t), \mu, s, N$, the application returns a numerical solution to $P_{k}(t)$. Accuracy is artificially made greater than that implied for the solvent - from $10^{-6}$ to $10^{-9}$ for absolute error and $10^{-3}$ to $10^{-5}$ for a relative error, respectively.

The input stream $\lambda(t)$, generally, is different every day, but some tendency and periodicity is noted. Measurements made in several non-attendance days in July 2017 show that roughly $50 \%(k=0.5)$ of the daily number of cars is between approximately 13:00 $\left(t_{0}=13 / 24\right)$ and 21:00 $\left(t_{2}=21 / 24\right)$ is about $50 \%(k=0.5)$ of the daily number of cars, with the peak being around 17:00 $\left(t_{1}=17 / 24\right)$, and the smallest between $4: 00$ and 6:00. The average daily number of cars for these few days is between 700 and $750(A \in[700 ; 750])$.

For modelling $\lambda(t)$, it is advisable to choose a relatively elementary function having a periodicity to reach the largest (lowest value) at peak times, and the integral sum at the busiest period is about $50 \%$ of the full-day flow. For a model, a Fourier line has been selected with three members $[17,18]$ :

$$
\lambda(t)=a_{0}+a_{1} \cos (2 \pi t)+b_{1} \sin (2 \pi t) .
$$

Coefficients $a_{0}, a_{1}, b_{1}$ are determined from the above comments:

1) $\int_{0}^{1} \lambda(t) d t=A-$ the sum of all cars of the day is $A$;

2) $\frac{d \lambda(t)}{d t}=0$, at $t=t_{1}$ - a necessary condition for the extreme in $t_{1}$;

3) $\int_{t_{0}}^{t_{2}} \lambda(t) d t=k A-$ in the peak interval $\left[t_{0}, t_{2}\right]$ a part of all $A$ cars equal to $k$ passes.

$1), 2$ ) and 3) result in the following system of linear algebraic equations with the unknown coefficients $a_{0}, a_{1}, b_{1}$ :

$\mid \begin{gathered}a_{0}=A \\ -a_{1} \sin \left(2 \pi t_{1}\right)+b_{1} \cos \left(2 \pi t_{1}\right)=0 \\ a_{0}\left(t_{2}-t_{0}\right)+a_{1} \frac{\sin \left(2 \pi t_{2}\right)-\sin \left(2 \pi t_{0}\right)}{2 \pi}+b_{1} \frac{\cos \left(2 \pi t_{0}\right)-\cos \left(2 \pi t_{2}\right)}{2 \pi}=k A\end{gathered}$

Figures 1 and 2 show the graphs of $\lambda(t)$ with specific meanings of the other parameters.

For the intensity of service from one channel $-\mu$, taking into account the service time of one car from one channel ( $7 \mathrm{~min})$, we receive for one day:

$\mu=24 \frac{60}{7}=205.714285$ automobiles per day per channel.

The integration of the system needs to be done not for a period of time but for a sufficient number of periods. In this way the probabilistic functions $P_{k}(t)$ begin to cling to their regular values. After multiple integration with different end times it was found out that only after 5-6 periods the functions $P_{k}(t)$ entered into a regular mode (for two adjacent periods, remain the same). Accuracy is also increased here, with integration being done for 20 periods, with the difference of all $P_{k}(t)$ in the last and penultimate periods being less than $10^{-8}$ for each $t$ [19].

The following graphs reflect the results of the system decision (2) at the following values:

$$
\begin{gathered}
A=730, k=0.5, t_{0}=\frac{13}{24}, t_{1}=\frac{17}{24}, t_{2}=\frac{21}{24}, \\
\lambda(t)=730-114.2318 \cos (2 \pi t)-426.3189 \sin (2 \pi t) \mu \\
=205.714285 \\
S=4 \text { (4 running servers) }
\end{gathered}
$$

Fig. 3 illustrates the probability of having exactly $k \in[0 ; 3]$ cars in the system. It is noteworthy that the most likely values for a small or zero number of cars are between 4:00 and 10:00.

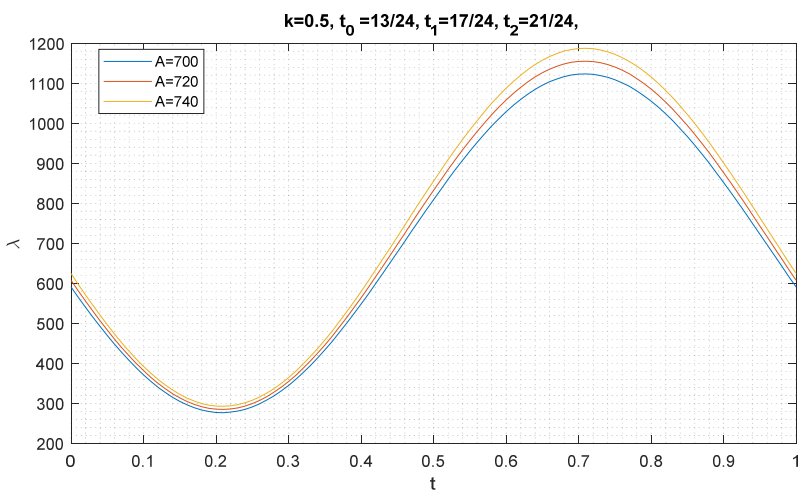

Fig. 1. Changing the coefficient $\lambda$ at different coefficient values A.

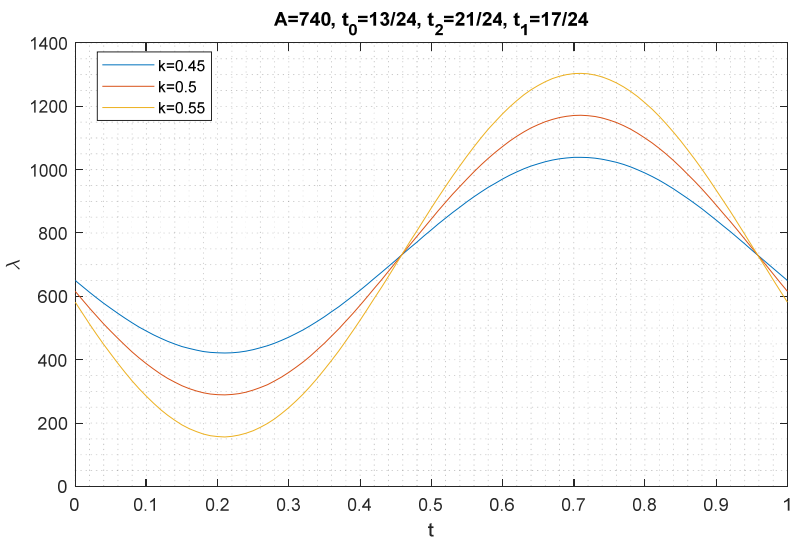

Fig.2. Changing the $\lambda$ coefficient at different coefficient $k$ values.

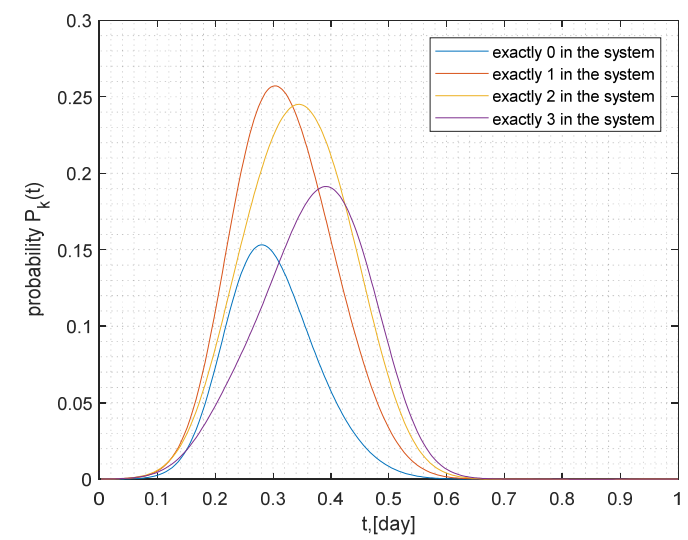

Fig. 3. Graph of the probability of having exactly $k \in[0 ; 3]$ automobiles in the system.

The probability of having exactly $k \in\{50,100,150\}$ automobiles in the system is presented in Fig. 4. Although the probabilities are small, the maximum number of automobiles is observed between 4 p.m. and 7 p.m. 
Fig. 5 shows the times when there are between 0 and 5 , between 6 and 15 , as well as between 16 and 30 automobiles. It is most likely to have between 0 and 5 automobiles at about 7:30 a.m.

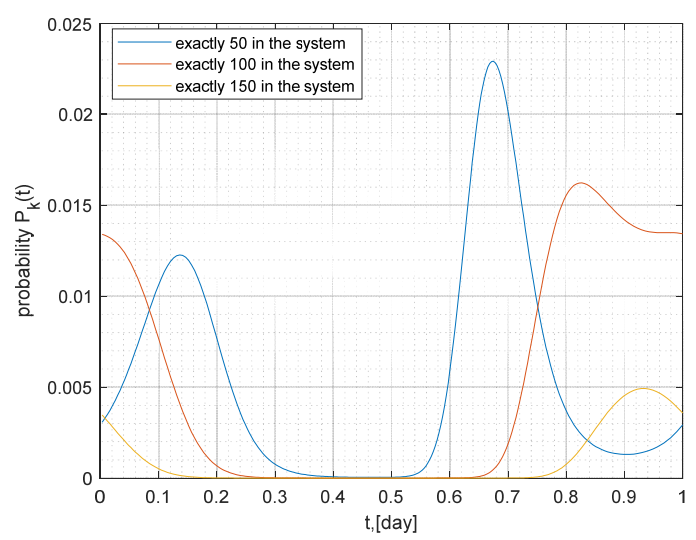

Fig.4. The probability of having exactly $k \in\{50,100,150\}$ automobiles in the system.

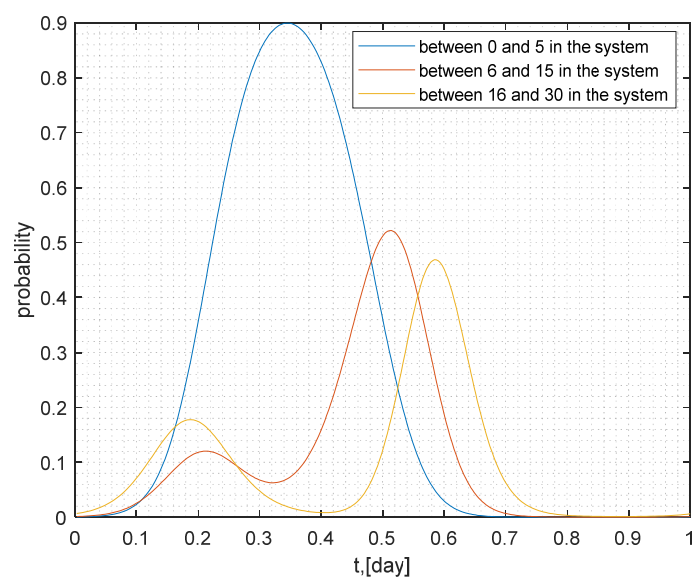

Fig.5. The probability of having between 0 and 5, between 6 and 15 , as well as between 16 and 30 automobiles.

Fig. 6 shows the likelihood to have between 91 and 120, between 121 and 170 and over 170 vehicles It is most likely to have the greatest number of cars in the system between 5 p.m. and 1 a.m.

The average number of queueing cars is given (Fig. 7). The highest number (over 100) is observed for about 21:30 minutes, and the smallest (less than 5) about 8:30.

Average vehicle downtime (on the abscissa are the hours) is shown in Fig. 8. The automobiles arriving at around 10 p.m. have to wait the longest time (over 160 min.), while those arriving at about 9 a.m. have the shortest time of waiting (under $5 \mathrm{~min}$.).

Research shows that when 4 pay desks are open constantly (12 teams), on a regular day (a day when the temperature is below 32 degrees), there is a marked imbalance in the main indicators of the system. The ineffective mode of operation of the system leads to the need of taking adequate measures to optimize it.

The observations made in July 2018, on regular days, as well as the data for the previous year, confirm that there is an increase in peak arrivals of cars between 4;30 p.m. and 6:30 p.m.

The smallest peak is observed between 4:00 and 6:00. With a permanent mode of operation of 4 cases (each with
3 teams on an 8-hour working day) there is a strong unevenness in the number of queuing cars as well as waiting times.

Between 20:00 and 0:00 there are more than 100 cars in the tail and a vehicle arriving during this interval has to wait over 160 minutes. And in the interval between 8:00 and 12:00 there are less than 5 cars in the tail, and a vehicle arriving during this interval, waits for 6-7 min.

Appropriate measures must be taken to reduce waiting times so that cars which arrived at a certain point have to wait for over 160 minutes, while others which arrived at another moment wait just 6-7 minutes, and regardless of the time of arrival, everyone would wait approximately the same time; this time should be as little as possible.

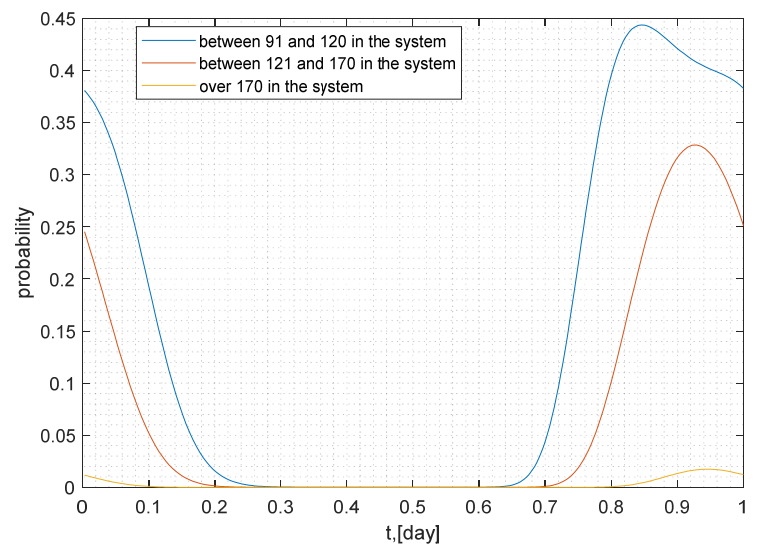

Fig. 6. The probability of having between 91 and 120, between 121 and 170 and over 170 vehicles.

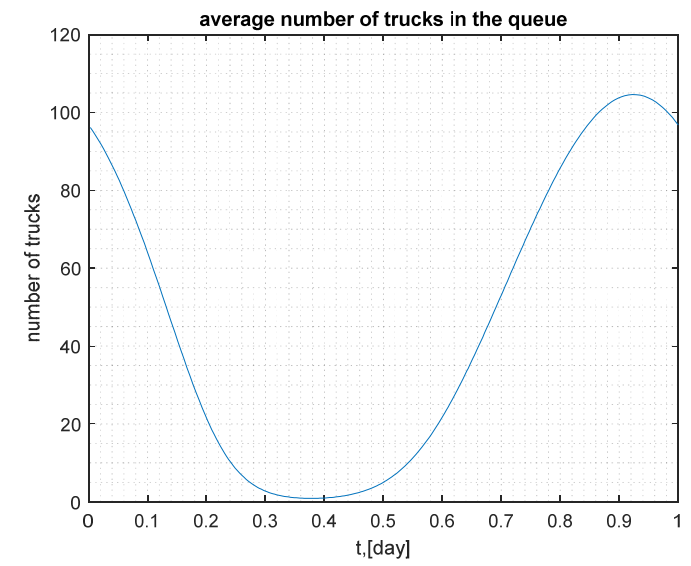

Fig. 7. Average number of cars queuing.

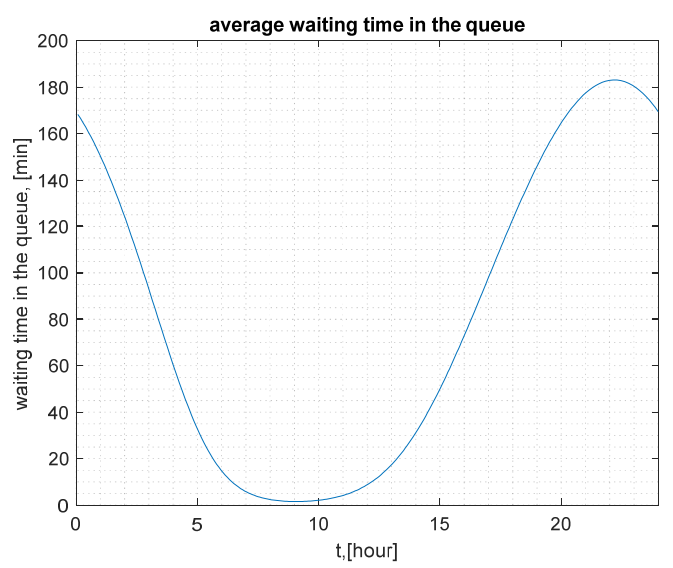

Fig.8. Average vehicle downtime per hour. 


\section{Conclusions}

1. The theoretical results obtained are intended to clarify the behavior of the system at any time of the day for periods of approximately equal non-stationary intensity (accuracy to constant) of arrival and service.

2. A way is proposed to approximate the input flow density by the most general average statistics.

3. A methodology is proposed for modelling and examination of the characteristics of a checkpoint/ checkpoints with similar non-stationary behavior, as well as those of checkpoints having any number of service units.

4. When system characteristics are unsatisfactory, the theoretical results will determine what adjustments in service intensity and / or number of channels are to be made for better performance. This would save time and problems of the "trial and error" type.

5. For the Ruse-Danube Bridge checkpoint, given the behavior of the average daily intensity, the "average queue duration" characteristic is synchronized sufficiently with the theoretical predictions (Table 1 and Fig. 8). With sufficient statistics and measurements (a sufficiently accurate approximated input and output of the intensity) for other checkpoints, it will provide theoretical characteristics that are close enough to the real ones.

6. Improvement can be achieved without incurring additional costs (e.g. for opening new positions that will make it possible for a fifth pay desk to work continuously). This can be done by organizing the working hours of the 12 teams in such a way that a fifth pay desk will work at specific times only, while only two or three pay checks will work in the remaining time. This organization of working hours will have the necessary effect for minimizing and regulating the waiting time. It would lead to a much more even mode of work for the system itself. The waiting time will be approximately the same regardless of the vehicles' arrival. The total average waiting time will also be reduced and this will take the burden off the transport companies themselves. They would plan a more optimal schedule, saving time and unnecessary costs. This would result in a release of funds that could be reinvested.

The study was supported by a contract of University of Ruse "Angel Kanchev", № BG05M2OP001-2.009-0011-C01, "Support for the development of human resources for research and innovation at the University of Ruse "Angel Kanchev". The project is funded with support from the Operational Program "Science and Education for Smart Growth 2014-2020" financed by the European Social Fund of the European Union.

\section{References}

1. R. Angelova, P. Stoyanov, N. Bunchyev, D. Grozev, Analysis of the traffic intensity in the border towns of Ruse and Vidin, Youth forums "Science, Technology, Innovation, Business" autumn 2017, Plovdiv, (2017)

2. Strategy for integrated border management of the Republic of Bulgaria 2014-2017 http://www.strategy.bg/PublicConsultations/View.as px?lang=bg-BG\&Id $=1281$
3. Strategy for development of the transport system of the republic of Bulgaria by 2020 March 2010 Sofia, (2010)

4. EUROSTAT (2015), Sustainable Development in the European Union: 2015 monitoring report of the EU sustainable development strategy, (EUROSTAT Statistical Books, 2015)

5. Bulgarian Helsinki Committee, Annual Report on Border Surveillance in 2017

6. B. Li, W. Li, Modeling and simulation of container terminal logistics systems using Harvard architecture and agent-based computing, Proceedings of the 2010 Winter Simulation Conference (2010)

7. C. Hsu, H. Shih, W. Wang, Applying RFID to reduce delay in import cargo customs clearance process, Computers \& Industrial Engineering, 57 (2009)

8. Exploring the possibilities for reducing the use of the TEN-T network in the cross-border region RomaniaBulgaria by optimizing the freight and passenger transport and development of a joint mechanism for intermodal connections support, http://www.adodunav.org/uploads/PDF/Brochure_B G_15.1.1.010-ilovepdf-compressed.pdf

9. J. Sztrik, Basic Queueing Theory, GlobeEdit, OmniScriptum GmbH, KG, Saarbrucken, Germany (2016)

10. M. Zukerman, Introduction to queueing theory and stochastic teletraffic models, EE Department, City University of Hong Kong (2016)

11. S. Ross, Introduction to probability models, (Elsevier, 2007)

12. L. Shampine, M. Reichelt, The MATLAB ODE Suite, SIAM Journal on Scientific Computing, 18, 1-22 (1997)

13. L. Norov, D. Akbarov, Customs - time for a change, Problems of Economic Transition, 52(2), 47-60 (2009)

14. A. Mitrofanova, Continuous times Markov chains. Poisson process. Birth and death process, (New York University, 2007)

15. N. Gautam, Analysis of queues: Methods and applications (CRC Press, 2012)

16. R. Markov, Transport corridors South-North in Southeastern Europe. Sustainable development of Lower Danube regions. Variant "Son - 2" of Rousse - Veliko Tarnovo Motorway, Sofia (2017)

17. M. Fitzmaurice, Optimization plan for freight movements at key commercial border crossings, Report compiled by Transport Logistics Consultants, August (2009)

18. H. Taha, Operations research, an introduction, New York (J. Wiley and Sons, 1993)

19. V. Todorov, I Georgiev, Optimization methods with MATLAB, (Avangard print, 2016) 